\title{
SGLT2 inhibitors: further evidence for heart failure with preserved ejection fraction as a metabolic disease?
}

\author{
Justin B. Echouffo-Tcheugui,, ${ }^{1}$ Sabra C. Lewsey, ${ }^{2}$ and Robert G. Weiss ${ }^{2}$
}

'Division of Endocrinology, Diabetes, and Metabolism and 'Division of Cardiology, Department of Medicine, Johns Hopkins University School of Medicine, Baltimore, Maryland, USA

\section{Heart failure with \\ preserved ejection fraction: therapeutic challenges}

Heart failure (HF) affects 6 million American adults, over half of whom have HF with preserved ejection fraction (HFpEF; ref. 1). HFpEF is a distinct syndrome from $\mathrm{HF}$ with reduced ejection fraction (HFrEF) and is now the predominant form of $\mathrm{HF}$ in older adults (2). Obesity, metabolic syndrome, hypertension, and diabetes predispose to $\mathrm{HFpEF}$, and its incidence is only expected to rise with an aging population and increasing burden of metabolic comorbidities (3). The prognosis for HFpEF is poor, and effective therapies to reduce adverse outcomes are lacking. Of note, conventional cardiovascular agents that improve outcomes in HFrEF patients, including vasodilators, venodilators, inotropes, and neurohormonal blockade, are largely ineffective in reducing primary outcomes in $\mathrm{HFpEF}$ trials (4). Metabolic interventions may offer a novel but largely unexplored approach to reduce adverse outcomes in HFpEF patients, considered the single largest unmet need in cardiovascular $(\mathrm{CV})$ medicine today (5).

Myocardial metabolic demands are the highest per gram of any organ in the body, and cardiac metabolism is altered in all animal models of $\mathrm{HF}$ and in patients with HF across etiologies (6). Whole body insulin resistance, impaired cardiac fatty acid oxidation, and reduced cardiac high-energy phosphate levels and creatine kinase energy reserve are among the most commonly observed myocardial metabolic hallmarks of experimental and human $\mathrm{HF}$ (6). On this background of HF as a metabolic disease, the results of the EMPEROR-Preserved trial were recently reported in The New England Journal of Medicine, and suggest that a metabolic approach with a sodium glucose cotransporter 2 inhibitor (SGLT2i) may answer this therapeutic challenge in HFpEF patients (7). Perhaps after years of neutral pharmacologic trials of conventional cardiovascular medications in HFpEF patients, it is apropos that the first medication to meet the primary endpoint in an HFpEF trial is an SGLT2i, arguably a "metabolic intervention."

\section{SCLT2is and clinical outcomes in $\mathrm{HF}$}

SGLT2is were initially developed as antidiabetic drugs because they inhibit glucose reabsorption in the proximal convoluted tubule of the kidney, leading to glucosuria and natriuresis. Reduced risk of CV death and HF hospitalizations in high-risk diabetic patients with SGLT2i use was first shown in the 2015 EMPA-REG trial of empagliflozin (8). Subsequent randomized trials of SGLT2is in HFrEF demonstrated significant and early reductions in the risk of HF hospitalization or CV death in patients with and without diabetes (9). Subgroup analyses of SGLT2is in HFrEF studies identified benefit even in the absence of volume overload, suggesting nondiuretic, possibly metabolic benefits of SGLT2is (10). Despite these results in HFrEF patients, trial data exclusively in HFpEF populations were lacking.

The recently reported EMPEROR-Preserved trial investigated the effects of empagliflozin exclusively among individuals with HFpEF (7). This phase III trial randomized 5,988 individuals (mean age 72 years, $45 \%$ women, $76 \%$ White participants) with class II-IV HF, an ejection fraction (EF) of $40 \%$ or greater, elevated NT-proBNP levels $(>300 \mathrm{pg} / \mathrm{mL}$, or $>900$ $\mathrm{pg} / \mathrm{mL}$ for patients with atrial fibrillation) to empagliflozin (10 mg daily) or placebo; the primary endpoint was a composite of CV death or hospitalization for HF. Over a 26-month period, the primary outcome was significantly reduced by empagliflozin versus placebo, with $21 \%$ and $29 \%$ relative reductions in the risks of the composite endpoint and of HF hospitalization, respectively. Empagliflozin did not decrease the overall and cause-specific death rates; thus, the benefit was mainly driven by reduction in HF hospitalizations. The empagliflozin effects were consistent across subgroups defined by age, diabetes status, obesity status, EF, and renal function. In addition, the benefits were rapidly apparent, with lower rates of HF hospitalization observed as early as 1 month after randomization in the empagliflozin group compared with the placebo group. However, in HFpEF patients, empagliflozin did not exhibit similar renal protective effects to those previously reported in individuals with HFrEF in the EMPEROR-Reduced trial (ClinicalTrials.gov NCT03057977), suggesting that renal effects are unlikely to be the primary mode of benefit in HFpEF. Although the use of empagliflozin was associated with more side effects (uncomplicated genital and urinary tract infections and hypotension) than placebo, there were similar rates of medication discontinuation in both study groups. The EMPEROR-Preserved results provide robust, novel evidence for the benefit of SGLT2is for treating HFpEF irrespective of diabetes status and will contribute to a major change in the management of HFpEF patients.

\section{Mechanism of SGLT2i protection in $\mathrm{HFpEF}$}

The mechanisms mediating the significant salutary effects of empagliflozin in HFpEF patients largely remain unclear, especially as SGLT2 expression in the healthy or failing heart is negligible (11), and SGLT2is do 
not bind to cardiac tissue (12). A number of molecular and cellular mechanisms have been suggested to account for the benefits of SGLT2is in HF and merit future investigation (as recently reviewed in ref. 13). The main putative explanatory mechanisms of the SGLT2i cardiac benefits include the following: (a) decreased ventricular loading conditions via reduced preload (secondary to natriuresis and osmotic diuresis) and afterload (secondary to reduction in blood pressure and improvement in vascular function), (b) improved cardiac metabolism and energetics, (c) a reduction in cytosolic $\mathrm{Ca}^{2+}$ and $\mathrm{Na}^{+}$in the myocardium through inhibition of the $\mathrm{Na}^{+} / \mathrm{H}^{+}$exchanger, (d) regression of left ventricular hypertrophy, (e) reduced necrosis and cardiac fibrosis, and (f) antiinflammatory and antioxidative effects via enhanced myocardial autophagy and reduced epicardial adipose tissue mass (including a reduction in its production of leptin; refs. 13, 14).

The early HF-related benefits in EMPEROR-Preserved (observed less than 1 month after randomization) may point to either improved loading conditions or metabolic mechanisms. However, the diuretic effects of SGLT2is are relatively short-lived in many studies, while SGLT2i benefit in HFrEF was reported to be independent of volume status (14). Although some of the cardiovascular effects of SGL$\mathrm{T} 2$ is could in theory be secondary to renal effects (13), no significant renal protection was observed in EMPEROR-Preserved (9). Indeed, SGLT2is may improve cardiac metabolism by increasing myocardial ATP production, possibly through enhanced ketone body availability (15). Circulating ketones can increase with SGLT2i treatment and provide an additional, advantageous myocardial carbon substrate for ATP production. Furthermore, ketone utilization is enhanced independently of diabetes status in end-stage $\mathrm{HF}$ patients (16). SGLT2is may also improve cardiac performance by affecting mitochondrial turnover, normalizing mitochondrial size and number (17), and also suppressing mitochondrial fission (18). The beneficial effects of SGLT2is in HFpEF may extend beyond the myocardium, with possible metabolic peripheral effects in skeletal muscle. Indeed, skeletal muscle metabolic abnormalities are prominent features of $\mathrm{HFpEF}$, and are closely linked to its hall- mark symptoms of exercise intolerance and fatigue (19). Metabolic alterations in skeletal muscle of HFpEF patients include reduced mitochondrial oxidative capacity, increased muscle fat content, and rapid high-energy phosphate decline during exercise (20).

A reasonable focus of future research exploring SGLT2i metabolic effects could include an assessment of cardiac and skeletal muscle substrate utilization and high-energy phosphate metabolism, given that energetic abnormalities are observed in animal models of $\mathrm{HF}$ and human $\mathrm{HF}$, including HFpEF. Human translational research on mechanisms of SGLT2i benefit in $\mathrm{HF}$ are critically needed and promise to fundamentally expand our understanding of HFpEF pathogenesis. Such an undertaking may identify additional therapeutic avenues for HFpEF.

\section{Implications for clinical practice}

Empagliflozin is the first pharmacologic therapy shown to reduce the composite of CV death or hospitalization in adults with symptomatic HFpEF, with or without diabetes mellitus (7). Although empagliflozin did not reduce $\mathrm{CV}$ death or all-cause mortality in HFpEF, HF hospitalizations are a particularly poor prognostic predictor and exact a high personal and societal toll. Additional insights regarding the impact of SGLT2is on cardiovascular mortality in HFpEF may be forthcoming from the pending DELIVER trial (ClinicalTrials.gov NCT03619213), a study of dapagliflozin in $\mathrm{HFpEF}$.

Because HFpEF populations are often heterogeneous, it is important to consider the predominant phenotype enrolled in the positive EMPEROR-Preserved trial. The population consisted of predominantly older adults, $45 \%$ women, with mean left ventricular EF of $54 \%$, mild $\mathrm{HF}$ symptoms, and borderline obesity, with an average body mass index of 30. Many participants were on renin-angiotensin system inhibitors (81\%), beta blockers (86\%), mineralocorticoid receptor antagonists (37\%), and statins (69\%). Only $2 \%$ were on sacubitril-valsartan. In contrast to the disproportionate burden of HF prevalence in African Americans, the enrollment of African Americans was less than 5\% (7). Thus, evaluation of outcomes in pooled data from EMPEROR-Preserved and future HFpEF clinical trials as well as in a real-world population of $\mathrm{HFpEF}$ patients with increased racial/ethnic diversity and varying severity of metabolic risk factors should be prioritized.

Nevertheless, meaningful experience previously gained from SGLT2is in HFrEF treatment should be helpful now in HFpEF management, affording clinicians added comfort with initiation, counseling regarding surveillance of genitourinary side effects, and clinical judgement in adjustment of background diuretic and glucose-lowering therapies. A practical point is that SGLT2is are relatively expensive medications whose affordability will depend on an individual's insurance coverage and socioeconomic status. Concomitant management of obesity, an important comorbid condition in many $\mathrm{HFpEF}$ patients, with appropriate lifestyle modification and cardioprotective therapy such as a glucagon-like peptide 1 receptor agonist may afford additional benefits (21). In the future, different HFpEF phenotypes will probably be considered to better elucidate which HFpEF patients are best suited to SGLT2i therapy.

In summary, the EMPEROR-Preserved trial represents a long-awaited breakthrough in the treatment of HFpEF. Empagliflozin reduced the primary composite endpoint of $\mathrm{CV}$ death or HF hospitalization in HFpEF patients and did so rapidly across demographic subgroups, including those without diabetes. After years of unsuccessful studies using conventional cardiovascular medications in $\mathrm{HFpEF}$, these new clinical findings with an SGLT2i offer a new treatment and further our understanding of HFpEF as a metabolic disease. Future studies are needed to define the specific mechanisms of cardiac protection and the role of metabolic reprograming therapy (using SGLT2is or other novel drugs) to reduce the disability, hospitalizations, and mortality from HFpEF.

\section{Acknowledgments}

JBET is funded by Clinical Scientist Development Award 2020094 from the Doris Duke Charitable Foundation, SCL by the NIH (AG063661-S1), and RGW by the NIH (HL61912, HL63030, HL156703, and AG063661) and the Clarence Doodeman Professorship in Cardiology at Johns Hopkins. 
Address correspondence to: Robert G. Weiss, Blalock 540, Johns Hopkins Hospital, 600 North Wolfe Street, Baltimore, Maryland 21287, USA. Phone: 410.955.1703; Email: rweiss@jhmi.edu.

1. Virani SS, et al. Heart disease and stroke statistics-2021 update: a report from the American Heart Association. Circulation. 2021;143(8):e254-e743.

2. Borlaug BA, Redfield MM. Diastolic and systolic heart failure are distinct phenotypes within the heart failure spectrum. Circulation. 2011;123(18):2014.

3. Dunlay SM, et al. Epidemiology of heart failure with preserved ejection fraction. Nat Rev Cardiol. 2017;14(10):591-602.

4. Borlaug BA. Evaluation and management of heart failure with preserved ejection fraction. Nat Rev Cardiol. 2020;17(9):559-573.

5. Butler J, et al. Developing therapies for heart failure with preserved ejection fraction: current state and future directions. JACC Heart Fail. 2014;2(2):97-112.

6. Tian R, et al. Unlocking the secrets of mitochondria in the cardiovascular system: path to a cure in heart failure-a report from the 2018 National Heart, Lung, and Blood Institute Workshop. Circulation. 2019;140(14):1205-1216.
7. Anker SD, et al. Empagliflozin in heart failure with a preserved ejection fraction. $N$ Engl J Med. 2021;385(16):1451-1461.

8. Zinman B, et al. Empagliflozin, cardiovascular outcomes, and mortality in type 2 diabetes. NEngl J Med. 2015;373(22):2117-2128.

9. Zannad F, et al. SGLT2 inhibitors in patients with heart failure with reduced ejection fraction: a meta-analysis of the EMPEROR-Reduced and DAPA-HF trials. Lancet. 2020;396(10254):819-829.

10. Packer M, et al. Empagliflozin in patients with heart failure, reduced ejection fraction, and volume overload: EMPEROR-Reduced trial. JAm Coll Cardiol. 2021;77(11):1381-1392.

11. Di Franco A, et al. Sodium-dependent glucose transporters (SGLT) in human ischemic heart: a new potential pharmacological target. Int $J$ Cardiol. 2017;243:86-90.

12. Ghezzi C, et al. Dapagliflozin binds specifically to sodium-glucose cotransporter 2 in the proximal renal tubule. J Am Soc Nephrol. 2017;28(3):802-810.

13. Lopaschuk GD, Verma S. Mechanisms of cardiovascular benefits of sodium glucose co-transporter 2 (SGLT2) inhibitors: a state-of-the-art review. JACC Basic to Transl Sci. 2020;5(6):632-644.

14. Pabel S, et al. SGLT2 inhibitors and their mode of action in heart failure-has the mystery been unravelled? Curr Heart Fail Rep. 2021;18(5):315-328.
15. Ferrannini E, et al. CV protection in the EMPA-REG OUTCOME trial: a thrifty substrate hypothesis. Diabetes Care. 2016;39(7):1108-1114.

16. Bedi KC Jr, et al. Evidence for intramyocardial disruption of lipid metabolism and increased myocardial ketone utilization in advanced human heart failure. Circulation. 2016;133(8):706-716.

17. Mizuno M, et al. Empagliflozin normalizes the size and number of mitochondria and prevents reduction in mitochondrial size after myocardial infarction in diabetic hearts. Physiol Rep. 2018;6(12):e13741.

18. Zhou H, et al. Empagliflozin rescues diabetic myocardial microvascular injury via AMPKmediated inhibition of mitochondrial fission. Redox Biol. 2018;15:335-346.

19. Fülster S, et al. Muscle wasting in patients with chronic heart failure: results from the studies investigating co-morbidities aggravating heart failure (SICA-HF). Eur Heart J. 2013;34(7):512-519.

20. Weiss K, et al. Fatigability, exercise intolerance, and abnormal skeletal muscle energetics in heart failure. Circ Heart Fail. 2017;10(7):e004129.

21. Khan MS, et al. Glucagon-like peptide 1 receptor agonists and heart failure: the need for further evidence generation and practice guidelines optimization. Circulation. 2020;142(12):1205-1218. 